\title{
Selection of reference genes for expression analysis in mouse models of acute alcoholic liver injury
}

\author{
SHENG WANG ${ }^{1-3}$, JIANQING WANG ${ }^{4}$ and XIONGWEN LV ${ }^{1-3}$ \\ ${ }^{1}$ School of Pharmacy; ${ }^{2}$ Institute for Liver Diseases of Anhui Medical University; \\ ${ }^{3}$ The Key Laboratory of Anti-inflammatory and Immune Medicines, Ministry of Education, \\ Anhui Medical University, Hefei, Anhui 230032; ${ }^{4}$ Department of Pharmacology, \\ The Second Hospital of Anhui Medical University, Hefei, Anhui 230601, P.R. China
}

Received September 29, 2017; Accepted February 8, 2018

DOI: $10.3892 / \mathrm{ijmm} .2018 .3527$

\begin{abstract}
Investigations of hepatic gene expression are crucial for determining the molecular factors involved in acute alcoholic liver injury. The results of liver molecular investigations may reveal etiologically important genomic alterations. Therefore, it is necessary to normalize gene expression data to identify stable genes, which may be used as a reference under different experimental conditions. The aim of the present study was to apply reverse transcription-quantitative polymerase chain reaction analysis and use analysis software to investigate the expression stability of candidate reference genes in hepatic tissues from mice with acute alcoholic liver injury. The acute alcoholic liver injury models were established by the intragastric administration of alcohol $(5 \mathrm{~g} / \mathrm{kg})$ in Imprinting Control Region mice. Total RNA was isolated from the mouse livers, following which the expression levels of seven reference genes, $\beta$-actin, glyceraldehyde 3-phosphate dehydrogenase (Gadph), glucuronidase $\beta$, hypoxanthine phosphoribosyltransferase 1 (Hprt1), 18S ribosomal RNA, TATA binding protein and $\beta-2$ microglobulin, were examined, and gene expression stability was assessed using the geNorm, NormFinder and BestKeeper tools. The geNorm analysis revealed that the gene with the lowest variability was Hprt1. Hprt1 and Gapdh were validated as the optimal reference gene pair in all samples from all groups. The NormFinder and BestKeeper results showed that Hprt1 was the most stable gene in all samples. Alcohol induces endoplasmic reticulum (ER) stress, causing changes in the expression levels of ER stress-associated genes. The stability of Hprtl was verified by the expression analysis of ER stress-associated genes, and gene expression levels in the
\end{abstract}

Correspondence to: Professor Xiongwen Lv, School of Pharmacy, Anhui Medical University, 81 Meishan Road, Hefei, Anhui 230032, P.R. China

E-mail: 1xw31288@aliyun.com

Key words: acute alcoholic liver injury, reference genes, reverse transcription-quantitative polymerase chain reaction, software analysis ethanol groups were upregulated, with a significant difference in expression, compared with those in the control group. Therefore, Hprt1 was selected as the most stable gene, and Hprt1 and Gapdh were determined to be the optimum gene pair in mouse models of acute alcoholic liver injury. The reliability of the Hprt1 gene was confirmed by expression analysis of ER stress-associated genes.

\section{Introduction}

Acute alcoholic liver injury refers to a period that may span several days, or periods of intermittent, repeated episodes of heavy drinking, which result in a spectrum of clinical signs and morphological changes, ranging from fatty liver (steatosis) to more severe forms of chronic liver injury, including fibrosis, cirrhosis and hepatocellular carcinoma (1-3). In addition, the increased prevalence of metabolic syndrome, and the combination of obesity, hypertension, dyslipidemia and hyperglycemia in a population, may present a risk factor to an increasing number of individuals with acute alcoholic liver injury manifestations (4-6). Liver injury and disease from excessive alcohol consumption have become important contributors to morbidity and mortality rates worldwide $(7,8)$.

The molecular events involved in acute alcoholic liver injury are complex, and altered gene expression ultimately orchestrates the integration of these distinct pathways in order to promote the response to alcoholic liver injury $(9,10)$. Previous studies have elucidated mechanisms that may be involved in the process of acute alcoholic liver injury, however, its molecular mechanism in terms of gene expression remains to be elucidated. Gene expression analysis in mouse models may provide important information on the molecular pathways involved in acute alcoholic liver injury, and reverse transcription-quantitative polymerase chain reaction (RT-qPCR) analysis has become the most important analytical tool for measuring gene expression due to its accuracy, sensitivity, specificity and reproducibility (11-13). However, the appropriate application of reverse transcription-quantitative polymerase chain reaction (RT-qPCR) analysis in comparative gene expression studies requires a rigorous normalization strategy to explain the technical variability among samples $(14,15)$. The use of reference genes as internal controls is the most common method 
of normalizing gene expression data (14). Consequently, it is important to select an appropriate reference gene for each experimental model. The selection of the reference gene(s) to use may be meaningful, and previous studies have demonstrated that a single common reference gene is not likely to be present and perform well for all tissue types, or under all physiological, pathological and experimental conditions (16). Furthermore, studies have demonstrated that the conventional use of a single reference gene for normalization may lead to relative errors. The use of multiple reference genes is currently considered to be the most effective approach for accurate normalization of data $(17,18)$.

The optimization of normalizing methods using reference genes has attracted increasing attention, leading to the development of several mathematical algorithms, including geNorm (19), NormFinder (20) and BestKeeper (21), which were developed to promote the evaluation of potential reference gene expression stability under different experimental conditions. Using these methods of statistical analysis, a number of reference genes have been selected for evaluation of their expression profiles under specific conditions (22).

The present study aimed to identify and evaluate the appropriate reference genes in a mouse model of ethanol-induced acute alcoholic liver injury. Using this model, the expression profiles of seven commonly used reference genes, $\beta$-actin (Actb), glyceraldehyde 3-phosphate dehydrogenase (Gadph), glucuronidase $\beta$ (Gusb), hypoxanthine phosphoribosyltransferase 1 (Hprt1), 18S ribosomal RNA (18S), TATA binding protein (Tbp) and $\beta-2$ microglobulin (B2m) were subsequently examined. Ethanol directly or indirectly leads to endoplasmic reticulum (ER) stress, causing changes in ER stress-associated gene expression (23). The stability of the selected reference gene was verified by expression analyses of ER stress-associated genes. It was observed that several genes commonly used to normalize qPCR data were not suitable for application as reference genes in acute alcoholic liver injury mouse models. The application of several mathematical algorithms under the set experimental conditions revealed that Hprt1 was the most stable gene, and that Hprt1 and Gapdh were the most appropriate gene pair to use in mouse models of acute alcoholic liver injury. The reliability of the selected reference genes was further confirmed by analyzing the expression of ER stress-associated genes.

\section{Materials and methods}

Chemicals and reagents. TRIzol ${ }^{\circledR}$ reagent was obtained from Invitrogen; Thermo Fisher Scientific, Inc. (Waltham, MA, USA). RNase-free DNase was purchased from Promega Corporation (Madison, WI, USA). A Reverse Transcription System kit was purchased from Promega Corporation. The Light Cycler 480 SYBR Green ${ }^{\circledR}$ I kit was obtained from Roche Diagnostics GmbH (Manheim, Germany). All other reagents were obtained from Sigma-Aldrich; Merck Millipore (Darmstadt, Germany), or as indicated in the specified methods.

Animals and treatments. Male Imprinting Control Region (ICR) mice (aged 8-10 weeks and weighing 28-30 g) were purchased from Beijing Vital River Laboratories Co., Ltd. (Beijing, China), whose foundation colonies were all introduced from Charles River Laboratories, Inc. (Yokohama, Japan). The animals were allowed free access to food and water at all times, and were maintained on a 12/12 h light/dark cycle with a controlled temperature $\left(20-25^{\circ} \mathrm{C}\right)$ and humidity $(50 \pm 5 \%)$ environment for 1 week prior to use. To identify the optimal reference gene in mouse models with ethanol-induced acute alcoholic liver injury, a total of 18 mice were divided into three groups ( $\mathrm{n}=6$ per group). The mice received ethanol $(5 \mathrm{~g} / \mathrm{kg})$ by intragastric administration. The control group received saline $(5 \mathrm{~g} / \mathrm{kg})$ by intragastric administration. At different time points ( 6 and $12 \mathrm{~h}$ ) following intragastric ethanol administration, the mice were weighed and sacrificed. All the mice were sacrificed following fasting for $14 \mathrm{~h}$, and liver and blood samples were collected. Liver tissue was collected and frozen immediately in liquid nitrogen for RT-qPCR analysis, or partially fixed in $4 \%$ paraformaldehyde for histological examination.

The present study was approved by the Association of Laboratory Animal Sciences and the Center for Laboratory Animal Sciences at Anhui Medical University (Hefei, China; permit no. 20150349). All procedures on animals conformed to the Guidelines for Humane Treatment set by the Association of Laboratory Animal Sciences and the Center for Laboratory Animal Sciences at Anhui Medical University.

Biochemical parameters and hepatic histology. Plasma was obtained from blood collected into tubes, after 2-6 h of storage at room temperature before centrifugation $(5,000 \mathrm{x} \mathrm{g}$, $10 \mathrm{~min}$ at $4^{\circ} \mathrm{C}$ ). The plasma alanine aminotransferase level was measured using commercial available kits (Nanjing Jiancheng Bioengineering Institute, Nanjing, China). Histological evaluation was performed using hematoxylin and eosin-stained tissue sections $(0.5 \times 0.5 \mathrm{~cm})$ and light microscopy. To quantify the extent of necrosis, the percentage of necrosis was estimated by measuring the necrotic area relative to the entire histological section. Analysis of the region was performed using NIH ImageJ software, version 1.44 (National Institutes of Health, Bethesda, MD, USA; http://rsb.info.nih.gov/ij/).

Terminal dUTP nick-end labeling (TUNEL) assay. For the detection of nuclear DNA strand breaks, the paraffin-embedded sections were stained with the TUNEL technique using an in situ apoptosis detection kit (Promega Corporation), according to the manufacturer's protocol. The sections were counterstained with hematoxylin. The TUNEL-positive cells were counted in 12 randomly selected fields from each slide at x200 magnification with a light microscope. The percentage of TUNEL-positive hepatocytes was analyzed in six liver sections from the six mice in each group.

Liver tissue collection and RNA isolation. Total RNA was isolated from the liver tissues of the saline- and ethanol-treated mice using TRIzol reagent. The liver samples were homogenized using $1.2 \mathrm{ml}$ TRIzol reagent per $50 \mathrm{mg}$ liver tissue. DNase I was used to digest and remove genomic DNA contaminants. The RNA purity was determined by measuring the absorbance at 260 and $280 \mathrm{~nm}$ with a microplate reader (ELX800, Bio-Tek Instruments, Inc., Winooski, VT, USA). The purity was verified at OD260/OD280 nm, and the ratios of 
Table I. Oligonucleotide sequences and sizes of primers.

\begin{tabular}{|c|c|c|}
\hline Gene & Sequence $\left(5^{\prime}-3^{\prime}\right)$ & (bp) \\
\hline \multirow[t]{2}{*}{ Actb } & Forward: GCTCTTTTCCAGCCTTCCTT & 92 \\
\hline & Reverse: CGGATGTCAACGTCACACTT & \\
\hline \multirow[t]{2}{*}{ Gapdh } & Forward: AGCCTCGTCCCGTAGACAA & 164 \\
\hline & Reverse: AATCTCCACTTTGCCACTGC & \\
\hline \multirow[t]{2}{*}{ Gusb } & Forward: AGCCTTCCTCTGCTCTGAAAC & 117 \\
\hline & Reverse: CTGCATCATATTTGGCGTTG & \\
\hline \multirow[t]{2}{*}{ Hprt1 } & Forward: CAAACTTTGCTTTCCCTGGT & 100 \\
\hline & Reverse: TCTGGCCTGTATCCAACACTTC & \\
\hline \multirow[t]{2}{*}{$18 \mathrm{~S}$} & Forward: TTGACGGAAGGGCACCACCAG & 130 \\
\hline & Reverse: GCACCACCACCCACGGAATCG & \\
\hline \multirow[t]{4}{*}{ Tbp } & Forward: GAAGAACAATCCAGACTAGC & 129 \\
\hline & AGCA & \\
\hline & Reverse: CCTTATAGGGAACTTCACATCA & \\
\hline & CAG & \\
\hline \multirow[t]{2}{*}{$\mathrm{B} 2 \mathrm{~m}$} & Forward: ATTCACCCCCACTGAGACTG & 193 \\
\hline & Reverse: TGCTATTTCTTTCTGCGTGC & \\
\hline
\end{tabular}

Actb, $\beta$-actin; Gapdh, glyceraldehyde 3-phosphate dehydrogenase; Gusb, glucuronidase $\beta$; Hprt1, hypoxanthine phosphoribosyltransferase 1; 18S, 18S ribosomal RNA; Tbp, TATA binding protein; B2m, $\beta$-2 microglobulin.

all samples ranged between 1.8 and 2.0. The RNA was stored at $-80^{\circ} \mathrm{C}$ until further analysis.

$R T$ - $q P C R$ analysis. The RNase-free DNase-treated total RNA $(1.0 \mu \mathrm{g}$ ) was reverse-transcribed with AMV (Promega Corporation). In order to improve the reverse transcription, random primers were used. The reactions were incubated at $37^{\circ} \mathrm{C}$ for $30 \mathrm{~min}, 65^{\circ} \mathrm{C}$ for $10 \mathrm{~min}$ and $42^{\circ} \mathrm{C}$ for $60 \mathrm{~min}$, and then diluted to a concentration of $0.5 \mu \mathrm{g} / \mu \mathrm{l}$. All cDNA was stored at $-20^{\circ} \mathrm{C}$ until required for the qPCR assay. The RT-qPCR analysis was performed with the Light Cycler 480 SYBR Green I kit using genetic-specific primers synthesized by Invitrogen; Thermo Fisher Scientific, Inc., as listed in Table I. The reaction mixture $(20 \mu \mathrm{l})$ consisted of $7 \mu \mathrm{l}$ of 10X buffer, $10 \mu \mathrm{l}$ of Mix (2X Taq DNA Polymerase, 2X PCR Buffer, 2X dNTP), $2 \mu \mathrm{l}$ of primer mix (forward and reverse primers) and $1 \mu \mathrm{l}$ of diluted cDNA. The amplification reactions were performed on a Light Cycler 480 instrument (Roche Diagnostics $\mathrm{GmbH}$ ), with an initial hold step $\left(95^{\circ} \mathrm{C}\right.$ for $\left.5 \mathrm{~min}\right)$ and 50 cycles of a three-step PCR $\left(95^{\circ} \mathrm{C}\right.$ for $15 \mathrm{sec}, 60^{\circ} \mathrm{C}$ for $15 \mathrm{sec}$ and $72^{\circ} \mathrm{C}$ for $\left.30 \mathrm{sec}\right)$. For the quantification of primers, a dissociation curve was drawn at the end of the run.

Statistical analysis. Seven candidate reference genes (Actb, Gapdh, Gusb, Hprt1, 18S, Tbp and B2m) were analyzed. The quantification cycle $(\mathrm{Cq})$ values were transformed into Raw Quantity (RQ) values via the $\Delta \mathrm{Cq}$ method $\left[R Q=2^{-(\Delta C q)}\right]$, $\Delta \mathrm{Cq}$ represents each corresponding $\mathrm{Cq}$ value - minimum $\mathrm{Cq}$ value (24). Two separate sets of independent samples from the control and treated mice were compared using an unpaired one-tailed t-test. Multiple-group comparisons were analyzed using one-way analysis of variance, followed by the Student-Newman-Keuls test. In all samples, $\mathrm{P}<0.05$ was considered to indicate a statistically significant difference. The data are expressed as the mean \pm standard deviation. In order to calculate the expression stability of the candidate reference genes, three validation mathematical algorithms were used, including BestKeeper (http://gene-quantification.com/ bestkeeper.html), which identifies the appropriate reference gene by paired correlation analysis of all pairs of candidate genes; geNorm (https://genorm.cmgg.be/), which calculates a gene normalization factor based on a pairwise comparison analysis, without considering the experimental conditions; and NormFinder (http://www.mdl.dk/publicationsnormfinder. $\mathrm{htm}$ ), which is based on a model selection method that enables estimation not only of the overall variation of the candidate normalization genes, but also of the variation between sample subgroups (20). The obtained RQ data were further analyzed with geNorm and NormFinder. BestKeeper analysis was based on the untransformed $\mathrm{Cq}$ values. For the rank of all candidate reference genes, the stability values from these three statistical algorithms were analyzed. The comparative Cq-method was used to determine the level of a target gene, normalized to a reference gene and relative to a calibrator $\left(2^{-\Delta \Delta C q}\right)$ using Lightcycler 480 software (Roche Diagnostics $\mathrm{GmbH}$; version $1.5 .0)(25,26)$.

\section{Results}

Ethanol treatment induces acute liver injury and hepatocyte necrosis. The characteristics of the mice administered with saline or $5 \mathrm{~g} / \mathrm{kg}$ ethanol intragastrically are listed in Table II. There was no statistically significant difference in mouse weights between the two groups. However, the ethanol-treated mice exhibited significantly elevated liver weights compared with those in the control group $(\mathrm{P}<0.05)$. In the ethanol group, the mice in the $12 \mathrm{~h}$ group exhibited a significant increase in hepatosomatic index (liver weight/body weight), compared with those in the $6 \mathrm{~h}$ group $(\mathrm{P}<0.05)$, whereas no significant difference in liver weight was found. The alanine aminotransferase activity was increased in the ethanol-treated group at different time points, and the activity in the ethanol $12 \mathrm{~h}$ group was significantly increased compared with that in the control group $(\mathrm{P}<0.05)$. Microscopic examination of the livers was performed to verify the damage caused by ethanol and to describe its typical histopathological characteristics. Characteristic hepatocyte necrosis was observed in the liver sections from the mice treated with ethanol (Fig. 1A). The area of necrosis was $\sim 28 \%$ at $12 \mathrm{~h}$ post-ethanol administration (Fig. 1B). Ethanol-induced hepatocyte death was determined using a TUNEL assay, and numerous TUNEL-positive cells were observed in the livers of the ethanol-treated mice (Fig. 1C and D). The ethanol $6 \mathrm{~h}$ group exhibited mild inflammatory infiltration and mild liver cell degeneration, whereas the tissue in the $12 \mathrm{~h}$ ethanol group exhibited severe infiltration and hepatocyte necrosis.

$R T-q P C R$ data analysis. Following data normalization, the $\mathrm{Cq}$ value was calculated for all PCR reactions. The Cq values for these seven reference genes were calculated for all mouse 
Table II. Characteristics of mice administered with saline or ethanol ( $5 \mathrm{~g} / \mathrm{kg}$ intragastrically).

Group

\begin{tabular}{|c|c|c|c|}
\hline Parameter & Control & Ethanol $6 \mathrm{~h}$ & Ethanol $12 \mathrm{~h}$ \\
\hline Weight (g) & $38.051 \pm 1.481$ & $39.183 \pm 1.682$ & $37.156 \pm 2.234$ \\
\hline Liver weight $(\mathrm{g})$ & $1.590 \pm 0.081$ & $1.714 \pm 0.072^{\mathrm{a}}$ & $1.763 \pm 0.181^{\mathrm{a}}$ \\
\hline Hepatosomatic index ${ }^{c}$ & $0.042 \pm 0.002$ & $0.044 \pm 0.001^{\mathrm{a}}$ & $0.047 \pm 0.003^{\mathrm{a}, \mathrm{b}}$ \\
\hline $\operatorname{ALT}(\mathrm{U} / \mathrm{l})$ & $37.021 \pm 4.342$ & $88.431 \pm 58.501$ & $184.046 \pm 55.663^{\mathrm{a}}$ \\
\hline
\end{tabular}

All data are expressed as the mean \pm standard deviation $(n=6) .{ }^{a} \mathrm{P}<0.05$, vs. control. ${ }^{\mathrm{b}} \mathrm{P}<0.05$, vs. ethanol 6 h. ${ }^{\mathrm{c}}$ Liver weight/body weight. ALT, alanine aminotransferase.
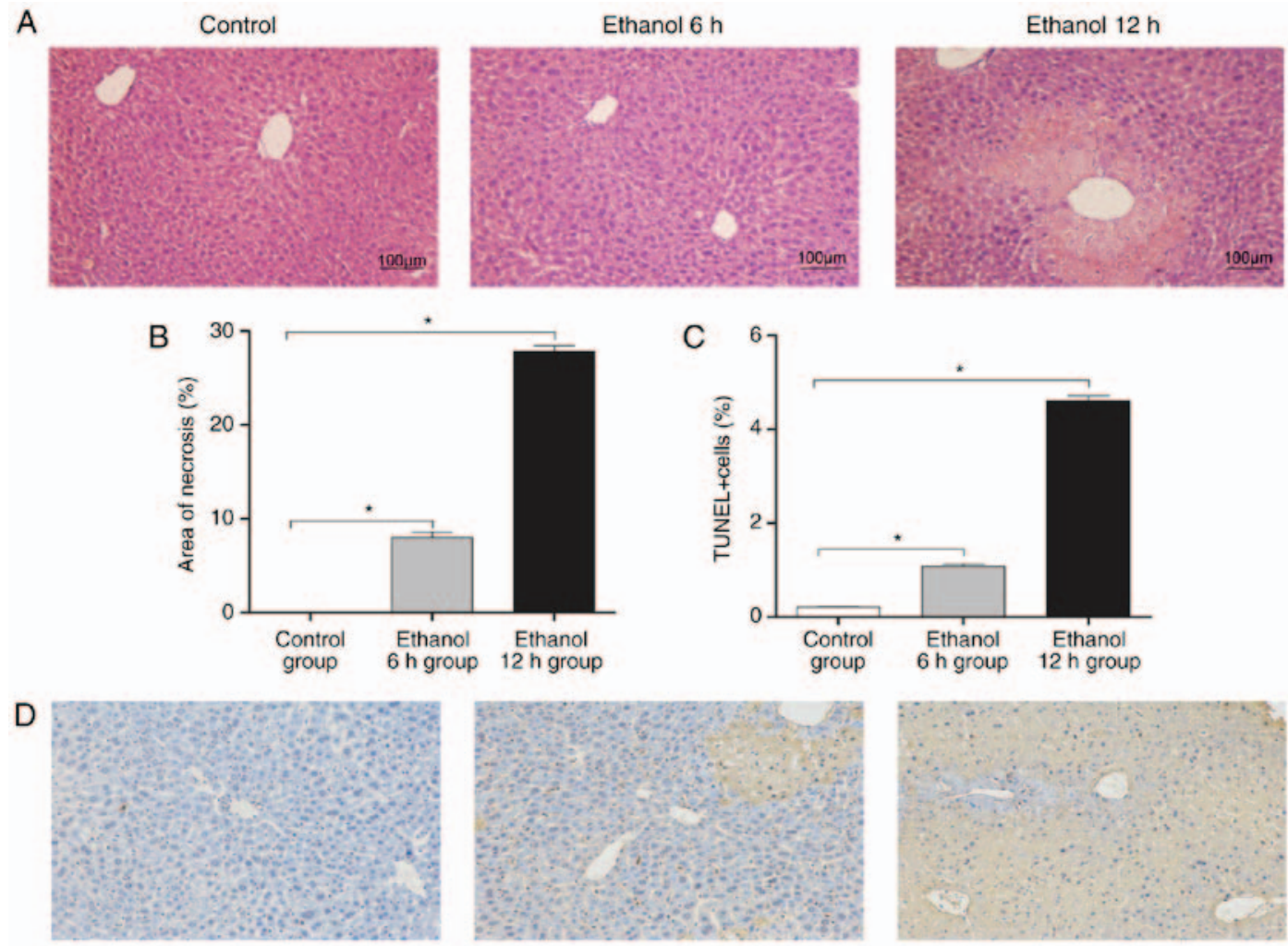

Figure 1. Ethanol treatment induces acute liver injury and hepatocyte death. The mice received an intragastric injection of ethanol (5 g/kg) for 6 or $12 \mathrm{~h}$ to develop acute liver injury. (A) Representative photomicrographs of liver histology (hematoxylin and eosin staining; magnification, x100). (B) Percentage area of necrosis in the liver was estimated. (C) Hepatocyte death was determined using a TUNEL assay and TUNEL ${ }^{+}$cells were analyzed. (D) Representative photomicrographs of TUNEL staining in different groups (magnification, $\mathrm{x} 200$ ). All data are expressed as the mean \pm standard deviation ( $\mathrm{n}=6$ ). ${ }^{*} \mathrm{P}<0.05$, compared with the control. TUNEL, terminal dUTP nick-end labeling.

livers sampled. The median $\mathrm{Cq}$ values of the seven reference genes ranged between 11.2 cycles for $18 \mathrm{~S}$ and 26.6 cycles for Tbp (Fig. 2A-G). Tbp and Gusb had the lowest expression levels, with median $\mathrm{Cq}$ values of 24-26 cycles. By contrast, $18 \mathrm{~S}$ and $\mathrm{B} 2 \mathrm{~m}$ had high expression levels with median $\mathrm{Cq}$ values ranging between 11 and 18 cycles. Actb, Gapdh and Hprt1 distributed intermediate expression levels with median $\mathrm{Cq}$ values between 20 and 23 cycles. Among the seven genes, Actb had the maximum range of expression at 3.2 cycles (19.6-22.8 cycles), whereas the minimum range of Gapdh was 1.3 cycles., The range of $\mathrm{Cq}$ values within each gene are shown in Fig. 2A-G. The extended vertical bars show standard the minimum and maximum values deviation of the mean in each gene. To an extent, it reflects the expression stability of each reference gene in the different groups.

Evaluation of the expression stability of the reference genes: geNorm analysis results. The present study first assessed the stability of expression for seven reference genes in the control, ethanol $6 \mathrm{~h}$, ethanol $12 \mathrm{~h}$ and all groups (Table III). The expression stability of the reference genes was analyzed using geNorm software. When all groups were analyzed, the genes examined exhibited expression stability measures ( $M$ values) between 0.11 (Hprt1) and 0.68 (Gusb). Hprt1 and Gapdh had the lowest $\mathrm{M}$ values, representing the most stable reference genes/gene pair in the liver in all samples. Reference gene 

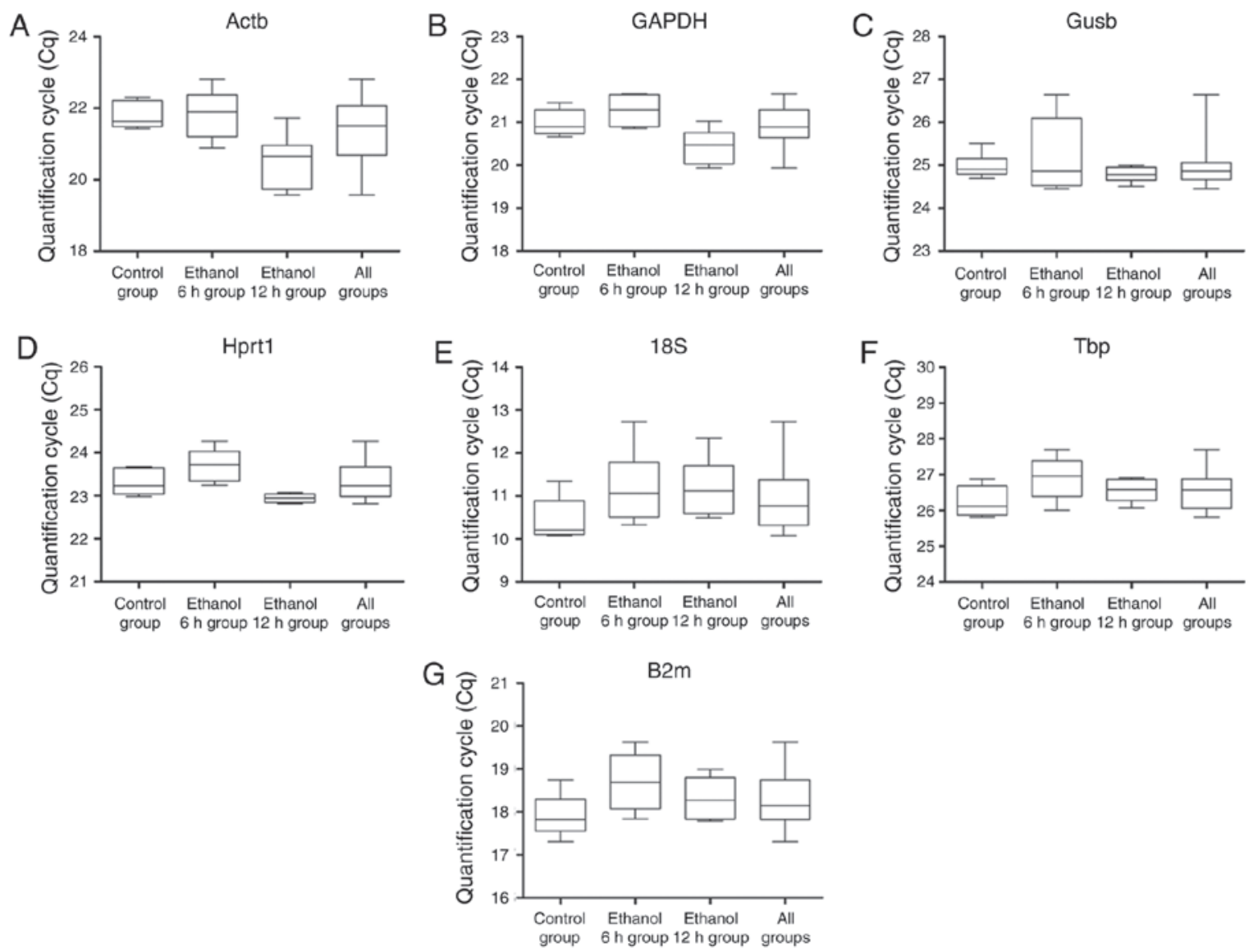

Figure 2. Reverse transcription-quantitative polymerase chain reaction Cq values of each candidate reference gene in four groups. The boxes indicate the range of $\mathrm{Cq}$ values within each gene. The central box represents the interquartile interval, the central lines indicate the median, and the extended vertical bars show the minimum and maximum standard deviation of the mean values for (A) Actb, (B) Gapdh, (C) Gusb, (D) Hprt1, (E) 18S, (F) Tbp and (G) B2m genes combined. Cq, quantification cycle; Actb, $\beta$-actin; Gapdh, glyceraldehyde 3-phosphate dehydrogenase; Gusb, glucuronidase $\beta$, Hprt1, hypoxanthine phosphoribosyltransferase 1; 18S, $18 \mathrm{~S}$ ribosomal RNA; Tbp, TATA binding protein; B2m, $\beta-2$ microglobulin.

stability analysis revealed that the gene with the lowest variability in all groups was Hprt1; 18S had the highest $\mathrm{M}$ value in the other three groups, with the exception of the assessment of 'all groups'. The result revealed that the least stable gene was $18 \mathrm{~S}$. The expression stability of the seven reference genes is shown in Fig. 3A-D

As shown in Fig. 4, the pairwise variations V2/3, V3/4, $\mathrm{V} 4 / 5, \mathrm{~V} 5 / 6$ and V6/7 were all lower than the limited value of 0.15 , which indicated that the combination of the two reference genes with the lowest $\mathrm{M}$ values in the experiments was sufficient for normalization. As all pairwise variations were $<0.15$, the above-mentioned observations remain valid, whether the data were analyzed for each experimental group, or in a single set grouping all data.

NormFinder analysis results. NormFinder also analyzed the stability values of the seven candidate reference genes. NormFinder is an Excel-based mathematical tool, which analyzes each sample set separately and also estimates inter-group variations in expression across different sample sets. NormFinder ranks the control genes on the basis of their stability value, where the lower stability value represents higher gene expression stability and vice versa. In the 'all groups' group and in the ethanol $12 \mathrm{~h}$ group, Hprt1 was identified as the most stable reference gene (Fig. 5 and Table III).
BestKeeper analysis results. Hprt1 was considered to be the top-ranked stable reference gene in all groups, whereas B2m and Gapdh were identified as the most stable internal reference genes in the control group, and in the ethanol $6 \mathrm{~h}$ and ethanol $12 \mathrm{~h}$ groups, respectively (Table III).

Differential gene expression associated with ER stress based on the selection of different reference genes. Ethanol can directly or indirectly lead to the occurrence of ER stress, and it may change the expression levels of glucose-regulated protein (GRP) 78 and of other genes $(27,28)$. It has been reported that the ER chaperone gene ER DNA J domain-containing protein 4 (ERdj4) is upregulated by ER stress (29). Protein disulfide isomerase (PDI) is a resident enzymatic chaperone and its expression is upregulated in ER stress (30). To demonstrate the importance of selecting appropriate reference genes as calibrators in ethanol treatment paradigms, ER stress-associated gene expression was normalized in the present study, with the most stable gene Hprt1 and the least stable gene $18 \mathrm{~S}$ as the reference genes analyzed by the three mathematical algorithms, geNorm, NormFinder and BestKeeper. The ER stress-associated genes included GRP78, C/EBP homologous protein (CHOP), GRP94, spliced X-box binding protein 1 (XBP1s) total XBP1 (XBP1t), ERdj4 and PDI. Using Hprt1 as the reference gene, the mRNA levels of GRP78, CHOP and PDI were significantly 
A

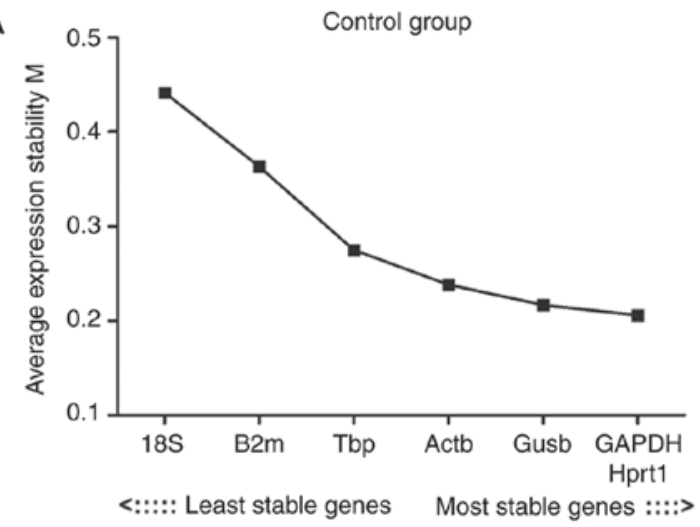

C

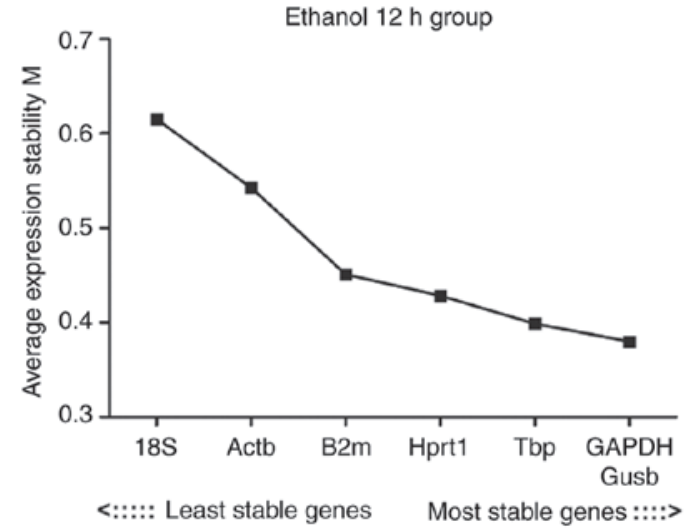

B

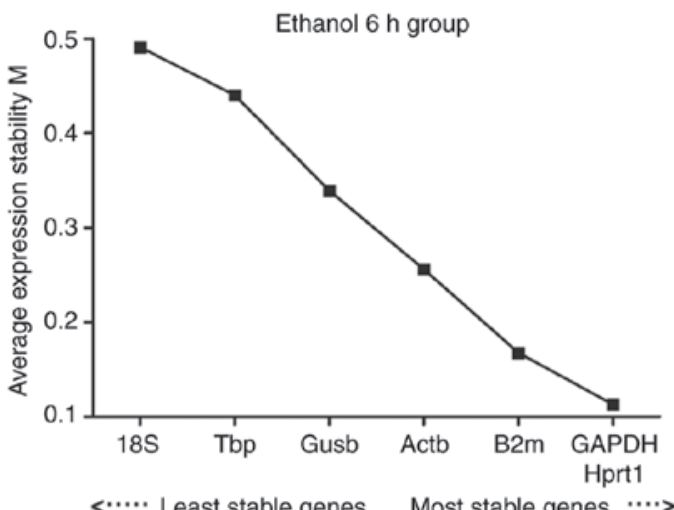

D

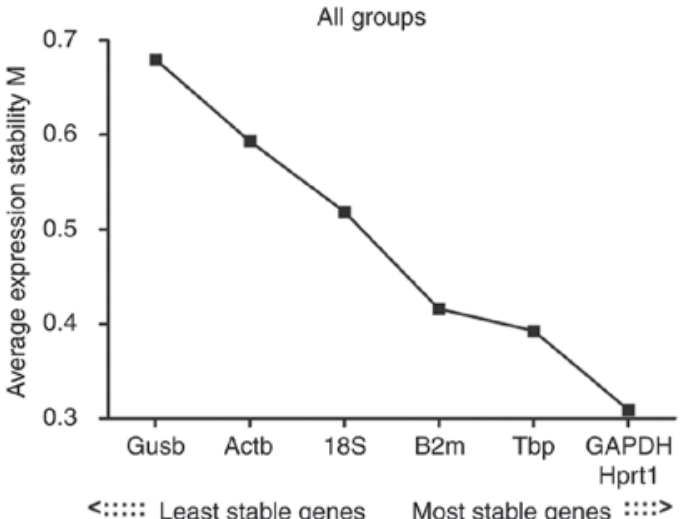

Figure 3. $\mathrm{M}$ value analysis of the seven reference genes calculated by geNorm. (A) Control group; (B) ethanol $6 \mathrm{~h}$ group; (C) ethanol $12 \mathrm{~h}$ group; (D) all groups. The least stable genes have high mean expression stability values (M); starting from the left, genes are ranked according to increasing expression stability, ending with the most stable genes on the right, with lower $\mathrm{M}$ values indicating more stable expression. M, expression stability; Actb, $\beta$-actin; Gapdh, glyceraldehyde 3-phosphate dehydrogenase; Gusb, glucuronidase $\beta$; Hprt1, hypoxanthine phosphoribosyltransferase 1; 18S, 18S ribosomal RNA; Tbp, TATA binding protein; $\mathrm{B} 2 \mathrm{~m}, \beta-2$ microglobulin.

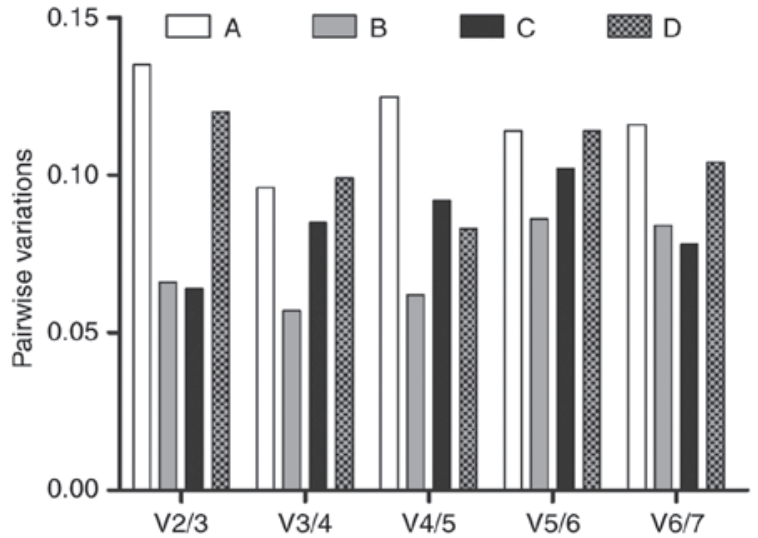

Figure 4. Vn analysis to determine the optimal number of control genes for accurate transcript normalization in all four experimental sets. geNorm was used to calculate pairwise variation $(\mathrm{Vn} / \mathrm{Vn}+1)$ for $\mathrm{NFn}$ and $\mathrm{NFn+1}$ to determine $(\mathrm{V}<0.15)$ the optimal number of reference genes. Data are plotted for the (A) control group, (B) ethanol $6 \mathrm{~h}$ group, (C) ethanol $12 \mathrm{~h}$ group and (D) all groups combined. Vn, pairwise variation; NF, normalization factor

increased in the livers of the ethanol $12 \mathrm{~h}$ group, compared with those in the control group, and the difference was statistically significant $(\mathrm{P}<0.05$; Fig. 6$)$. The relative expression levels of the remaining genes, ERdj4, GRP94 and XBP1t, exhibited a gradually increasing trend in the control to the ethanol groups. Using $18 \mathrm{~S}$ as the reference gene, no statistically significant differences in gene expression were found between the ethanol and control groups. These results suggested that Hprt1 offers an advantage as a reference gene in mouse models of acute alcoholic liver injury.

\section{Discussion}

With regards to endogenously expressed reference genes, the appropriate selection of an individual or of a pair of reference genes is crucial for the quantification of gene expression under specific conditions. Similarly, the establishment of well-characterized animal models is also crucial to fully understand the condition represented by the model. RT-qPCR analysis of gene expression is the most common method for examining relevant changes in gene regulation, and provides rapid and consistent results. The use of reference genes is generally considered to be the most reliable method of normalizing qPCR data and reducing possible errors in the quantification of gene expression (31); however, their utility requires experimental validation for particular experimental designs (32). The consistent expression of the reference gene is crucial to ensure correct analysis of the experimental results. The inadequate selection of reference genes may lead to an erroneous analysis and interpretation of relative expression, particularly when there are marginal variations in transcription levels between different individuals, sample groups and experimental conditions $(33,34)$. Therefore, one of the key points in validation is 


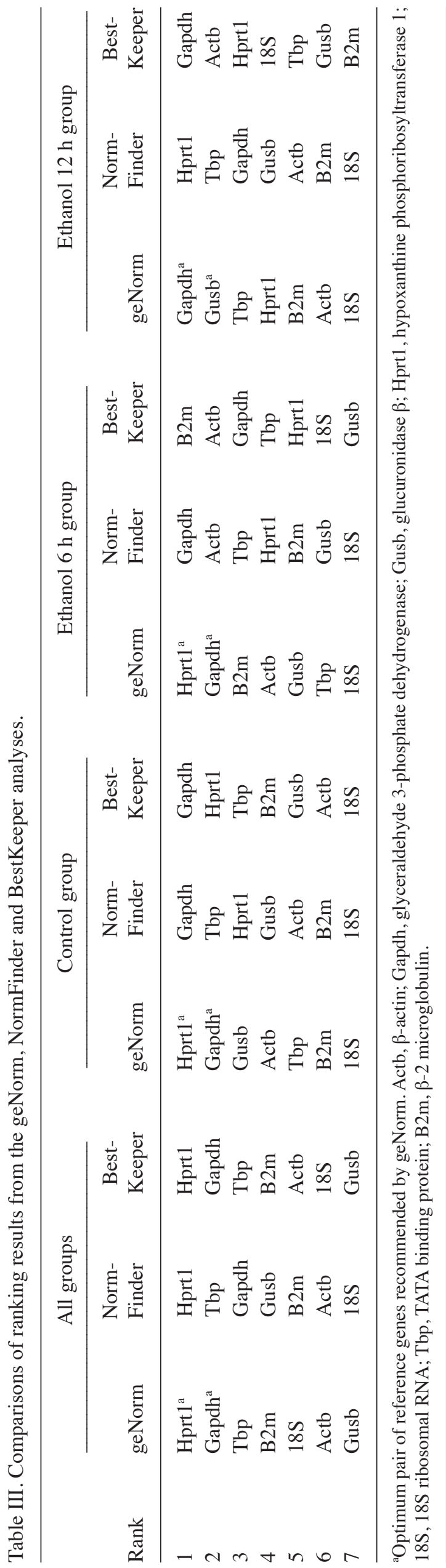

to select appropriate reference genes for data normalization in gene expression analyses. In the present study, seven reference genes were examined, which were selected due to their common use in previously published reports on the liver and other type of tissues. ER stress-associated gene expression measurements can be demonstrably affected by the selection of reference genes. Therefore, it is necessary to select appropriate reference genes for the quantitation of genes via $\mathrm{qPCR}$ in acute alcoholic liver injury.

The commonly used programs, geNorm, NormFinder and BestKeeper, estimated the most stable reference genes. The ranking of the reference genes examined by the three programs varied marginally (Table III), however, this was not unexpected as the different programs rely on distinct mathematical approaches and analytical principles (35). The geNorm algorithm calculates an average expression stability $M$ value for each gene from a set of reference genes used in the analysis. NormFinder identifies stable expression genes in a set of candidate normalization genes based on a mathematical model that can estimate the intra- and inter-group variations of the sample set. BestKeeper is an Excel-based tool to assist in selecting the optimal reference genes following calculation of variables. The results in the present study were based on these three mathematical algorithms, which confirmed that Hprt1 was the reference gene with the most stable expression level among the seven candidate genes, regardless of the different mouse models. In addition, $18 \mathrm{~S}$ was identified as the least stable reference gene. However, several experiments using qPCR analysis have selected other reference genes, for example 18S and Actb, even when it has been reported that these common reference genes are not stably expressed under variable experimental conditions $(36,37)$. It has been previously reported that $18 \mathrm{~S}$ as a reference gene shows the least expression stability $(38,39)$.

Hprt1 is a common reference gene for normalizing relative expression values in qPCR analysis $(40,41)$. Gapdh is also reliable as a reference gene for quantitative gene expression analysis under experimental conditions (42). Although there is a possibility that there are more appropriate reference genes other than those analyzed in the present study, the results confirmed that Hprt1 and Gapdh exhibited reliable and stable gene expression, compared with other more commonly used reference genes, including 18S and Actb (Figs. 3 and 5; Table III).

Following estimation of the $\mathrm{M}$ value, geNorm calculates the minimum number of genes necessary for an appropriate normalization. NormFinder, provides the optimal reference gene pairs for normalization and suggests that multiple reference genes only be used when a single stable gene cannot be selected (43). Therefore, Hprt1, a reference gene selected by NormFinder and geNorm, may be used as a suitable reference gene in mouse models of acute alcoholic liver injury.

Ethanol induces ER stress, causing changes in the expression levels of ER stress-associated genes. The stability of the selected reference genes was verified by expression analysis of ER stress-associated genes. Using the most stable reference gene, Hprt1, and the least stable gene, 18S, each identified by the three statistical algorithms, the results demonstrated that, compared with the control group and using Hprtl as the reference gene, ER stress-associated gene expression was upregulated in the ethanol groups with a statistically significant difference; 

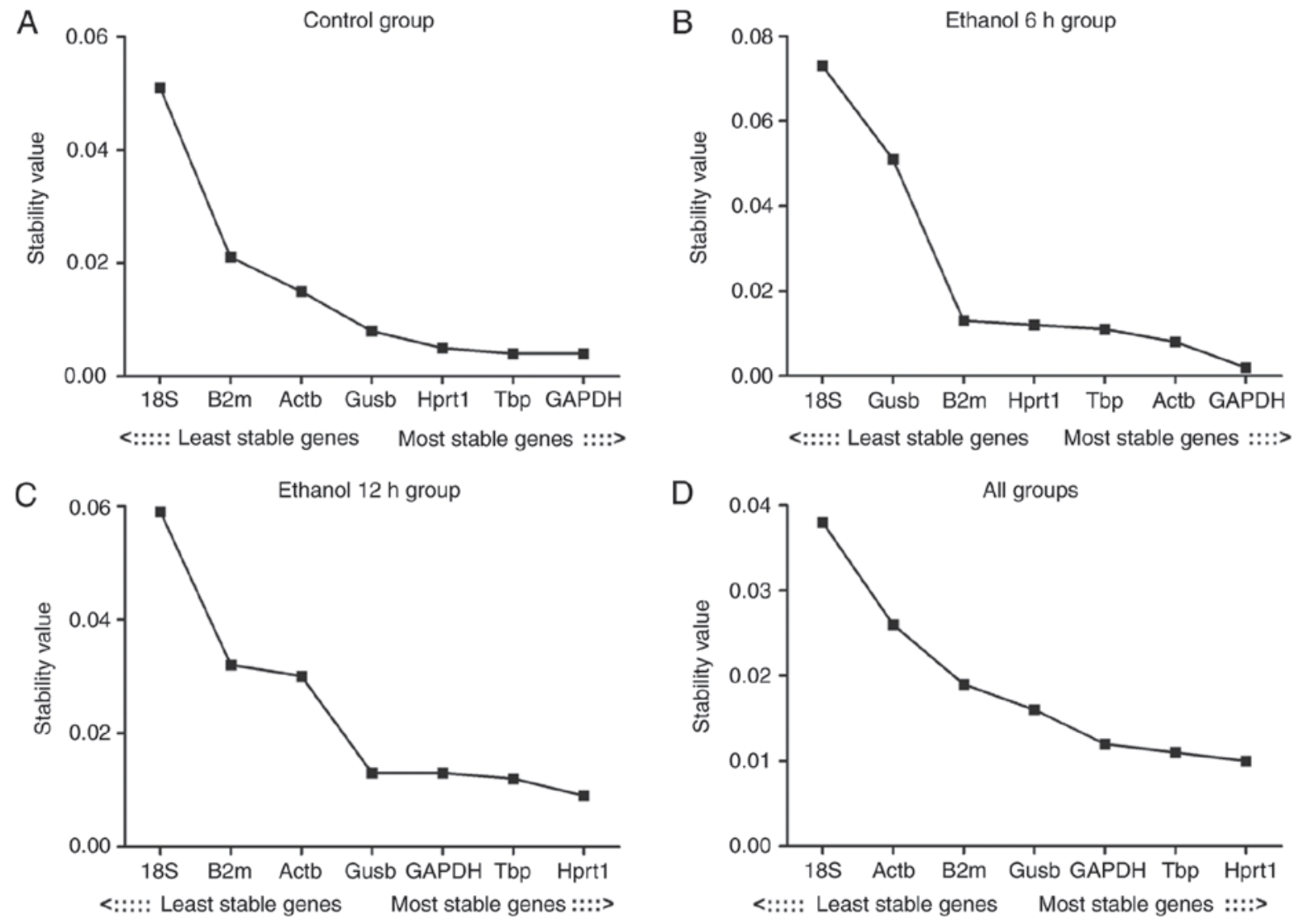

Figure 5. Stability estimation for seven reference genes by NormFinder. (A) Control group; (B) ethanol $6 \mathrm{~h}$ group; (C) ethanol $12 \mathrm{~h}$ group; (D) all groups. The least stable gene with high stability values starts from the left, ending with the most stable genes on the right. Lower stability values indicate more stable expression. Actb, $\beta$-actin; Gapdh, glyceraldehyde 3-phosphate dehydrogenase; Gusb, glucuronidase $\beta$, Hprt1, hypoxanthine phosphoribosyltransferase 1; $18 \mathrm{~S}$, 18S ribosomal RNA; Tbp, TATA binding protein; B2m, $\beta-2$ microglobulin.
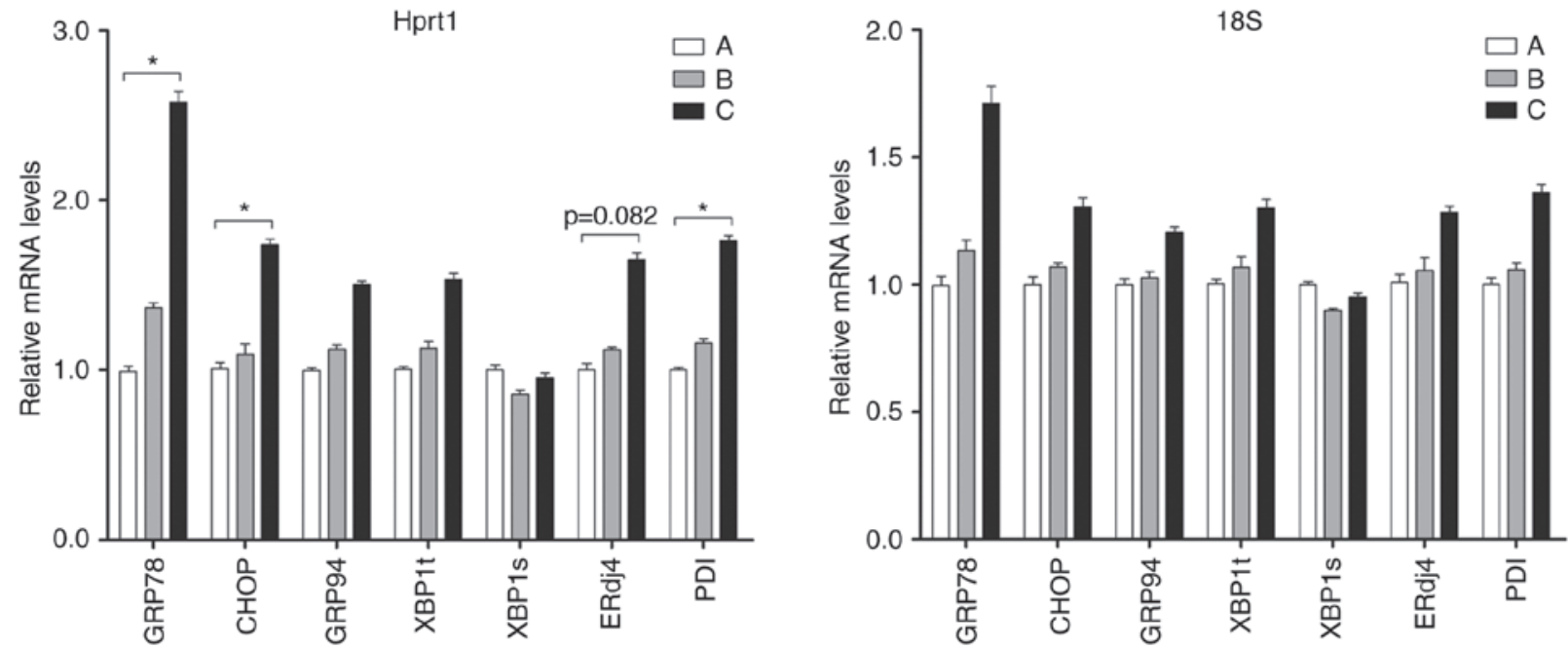

Figure 6. Levels of different ER stress-associated genes. Hprt1 and $18 \mathrm{~S}$ were used as reference genes for reverse transcription-quantitative polymerase chain reaction analysis. (A) Control group; (B) ethanol $6 \mathrm{~h}$ group; (C) ethanol $12 \mathrm{~h}$ group. All data are expressed as the mean \pm standard deviation ( $\mathrm{n}=6$ ). "P<0.05, compared with the control. ER, endoplasmic reticulum; Hprt1, hypoxanthine phosphoribosyltransferase 1; 18S, 18S ribosomal RNA; GRP78, glucose-regulated protein 78; CHOP, C/EBP homologous protein; GRP94, glucose-regulated protein 94; XBP1a, spliced X-box binding protein 1; XBP1t, total XBP1; ERdj4, ER DNA J domain-containing protein 4; PDI, protein disulfide isomerase

whereas the use of $18 \mathrm{~S}$ as the reference gene revealed no statistically significant difference between the ethanol and control groups (Fig. 6). These results suggested that Hprt1 is a suitable reference gene in mouse models of acute alcoholic liver injury.
Therefore, selecting a commonly used reference gene without first evaluating its stability may result in incorrect normalization and altered quantification of target gene expression, affecting the interpretation of the results. 
In conclusion, the suitable selection of reference genes is a crucial step in the characterization of any animal model. Seven commonly used reference genes were examined in the present study to identify their stability in the livers of mice with acute alcoholic liver injury. The reliability of the selected reference genes was further verified by expression analysis of ER stress-associated genes. The results of the present study demonstrated that the advantages of Hprt1 in normalizing target gene expression make it a feasible method for the accurate quantification of gene expression associated with acute alcoholic liver injury. These findings may assist in subsequent investigations of gene expression using this mouse model. However, only the expression stabilities of select reference genes in ICR mice with acute alcoholic liver injury were evaluated in the present study. Whether the selected reference genes are also suitable for other commonly used mouse strains, for example $\mathrm{C} 57 \mathrm{BL} / 6$, remains to be elucidated. In order to optimize the gene expression analysis in this mouse model, the stability of the reference gene in other mouse strains requires assessment in subsequent investigations to further elucidate the molecular mechanisms involved in mouse models of acute alcoholic liver injury.

\section{Acknowledgements}

Not applicable.

\section{Funding}

This study was supported by the National Science Foundation of China (grant nos. 81270498 and 81400643) and the 2016 Annual Leading Talent Introduction and Cultivation Project in Universities (grant no. gxbjZD2016032).

\section{Availability of data and materials}

The analyzed data sets generated during the study are available from the corresponding author on reasonable request.

\section{Authors' contributions}

All authors have read and approved the contents of this manuscript for publication. XL provided experimental animal models. JW analyzed and interpreted the mouse models data. SW performed the histological examination of the liver and was a major contributor in writing the manuscript.

\section{Ethics approval and consent to participate}

The study was approved by the Association of Laboratory Animal Sciences and the Center for Laboratory Animal Sciences at Anhui Medical University (permit no. 20150349). All procedures on animals conformed to the Guidelines for Humane Treatment set by the Association of Laboratory Animal Sciences and the Center for Laboratory Animal Sciences at Anhui Medical University.

\section{Consent for publication}

Not applicable.

\section{Competing interests}

No conflict of interest exists in the submission of this manuscript.

\section{References}

1. Massey VL and Arteel GE: Acute alcohol-induced liver injury. Front Physiol 3: 193, 2012.

2. Gonçalves JL, Lacerda-Queiroz N, Sabino JFL, Marques PE, Galvão I, Gamba CO, Cassali GD, de Carvalho LM, da Silva E Silva DA, Versiani A, et al: Evaluating the effects of refined carbohydrate and fat diets with acute ethanol consumption using a mouse model of alcoholic liver injury. J Nutr Biochem 39: 93-100, 2016.

3. Sugimoto K and Takei Y: Pathogenesis of alcoholic liver disease. Hepatol Res 47: 70-79, 2017.

4. Chang B, Xu MJ, Zhou Z, Cai Y, Li M, Wang W, Feng D, Bertola A, Wang H, Kunos G and Gao B: Short- or long-term high-fat diet feeding plus acute ethanol binge synergistically induce acute liver injury in mice: An important role for CXCL1. Hepatology 62: 1070-1085, 2015.

5. Song BJ, Abdelmegeed MA, Henderson LE, Yoo SH, Wan J, Purohit V, Hardwick JP and Moon KH: Increased nitroxidative stress promotes mitochondrial dysfunction in alcoholic and nonalcoholic fatty liver disease. Oxid Med Cell Longev 2013: 781050, 2013.

6. Fujita $\mathrm{N}$ and Takei $\mathrm{Y}$ : Alcohol consumption and metabolic syndrome. Hepatol Res 41: 287-295, 2011.

7. Zhang JJ, Meng X, Li Y, Zhou Y, Xu DP, Li S and Li HB: Effects of melatonin on liver injuries and diseases. Int J Mol Sci 18: E673, 2017.

8. Chacko KR and Reinus J: Spectrum of alcoholic liver disease. Clin Liver Dis 20: 419-427, 2016.

9. Tsuchiya M, Ji C, Kosyk O, Shymonyak S, Melnyk S, Kono H, Tryndyak V, Muskhelishvili L, Pogribny IP, Kaplowitz N and Rusyn I: Interstrain differences in liver injury and one-carbon metabolism in alcohol-fed mice. Hepatology 56: 130-139, 2012.

10. Halsted CH: B-Vitamin dependent methionine metabolism and alcoholic liver disease. Clin Chem Lab Med 51: 457-465, 2013.

11. Nolan T, Hands RE and Bustin SA: Quantification of mRNA using real-time RT-PCR. Nat Protoc 1: 1559-1582, 2006.

12. Derveaux S, Vandesompele J and Hellemans J: How to do successful gene expression analysis using real-time PCR. Methods 50: 227-230, 2010.

13. Ballester M, Cordon R and Folch JM: DAG expression: High-throughput gene expression analysis of real-time PCR data using standard curves for relative quantification. PloS One 8: e80385, 2013.

14. Lardizabal MN, Nocito AL, Daniele SM, Ornella LA, Palatnik JF and Veggi LM: Reference genes for real-time PCR quantification of microRNAs and messenger RNAs in rat models of hepatotoxicity. PLoS One 7: e36323, 2012.

15. Hernández AH, Curi R and Salazar LA: Selection of reference genes for expression analyses in liver of rats with impaired glucose metabolism. Int J Clin Exp Pathol 8: 3946-3954, 2015.

16. Xu XY, Shen YB, Fu JJ, Lu LQ and Li JL: Determination of reference microRNAs for relative quantification in grass carp (Ctenopharyngodon idella). Fish Shellfish Immunol 36: 374-382, 2014.

17. Matouskova P, Bartikova H, Bousova I, Hanusova V, Szotakova B and Skalova L: Reference genes for real-time PCR quantification of messenger RNAs and microRNAs in mouse model of obesity. PLoS One 9: e86033, 2014.

18. Castonguay Y, Michaud J and Dubé MP: Reference genes for RT-qPCR analysis of environmentally and developmentally regulated gene expression in alfalfa. Am J Plant Sci 6: 132-143, 2015.

19. Vandesompele J, De Preter K, Pattyn F, Poppe B, Van Roy N, De Paepe A and Speleman F: Accurate normalization of real-time quantitative RT-PCR data by geometric averaging of multiple internal control genes. Genome Biol 3: RESEARCH0034, 2002.

20. Andersen CL, Jensen JL and Ørntoft TF: Normalization of real-time quantitative reverse transcription-pcr data: A model-based variance estimation approach to identify genes suited for normalization, applied to bladder and colon cancer data sets. Cancer Res 64: 5245-5250, 2004. 
21. Pfaffl MW, Tichopad A, Prgomet $C$ and Neuvians TP Determination of stable housekeeping genes, differentially regulated target genes and sample integrity: BestKeeper-Excel-based tool using pair-wise correlations. Biotechnol Lett 26: 509-515, 2004.

22. Tatsumi K, Ohashi K, Taminishi S, Okano T, Yoshioka A and Shima M: Reference gene selection for real-time RT-PCR in regenerating mouse livers. Biochem Biophys Res Commun 374 106-110, 2008

23. Ji C: New insights into the pathogenesis of alcohol-induced ER stress and liver diseases. Int J Hepatol 2014: 513787, 2014.

24. Schmittgen TD and Livak KJ: Analyzing real-time PCR data by the comparative C (T) method. Nat Protoc 3: 1101-1108, 2008

25. Ferlini A and Rimessi P: Exon skipping quantification by real-time PCR. Methods Mol Biol 867: 189-199, 2012.

26. Rao X, Huang $X$, Zhou $Z$ and Lin $X$ : An improvement of the $2^{\wedge}$ (-delta delta CT) method for quantitative real-time polymerase chain reaction data analysis. Biostat Bioinforma Biomath 3 : 71-85, 2013.

27. Ji C and Kaplowitz N: ER stress: Can the liver cope? J Hepatol 45: 321-333, 2006

28. Malhi $\mathrm{H}$ and Kaufman RJ: Endoplasmic reticulum stress in liver disease. J Hepatol 54: 795-809, 2011.

29. Lai CW, Otero JH, Hendershot LM and Snapp E: ERdj4 protein is a soluble endoplasmic reticulum (ER) DnaJ family protein that interacts with ER-associated degradation machinery. J Biol Chem 287: 7969-7978, 2012.

30. Wang SB, Shi Q, Xu Y, Xie WL, Zhang J, Tian C, Guo Y, Wang K, Zhang BY, Chen C, et al: Protein disulfide isomerase regulates endoplasmic reticulum stress and the apoptotic process during prion infection and PrP mutant-induced cytotoxicity. PLoS One 7: e38221, 2012.

31. Peletto S, Bertuzzi S, Campanella C, Modesto P, Maniaci MG, Bellino C, Ariello D, Quasso A, Caramelli M and Acutis PL: Evaluation of internal reference genes for quantitative expression analysis by real-time PCR in ovine whole blood. Int J Mol Sci 12 : 7732-7747, 2011.

32. Bustin SA, Benes V, Garson JA, Hellemans J, Huggett J, Kubista M, Mueller R, Nolan T, Pfaffl MW, Shipley GL, et al: The MIQE guidelines: Minimum information for publication of quantitative real-time PCR experiments. Clin Chem 55: 611-622, 2009.
33. Lacerda AL, Fonseca LN, Blawid R, Boiteux LS, Ribeiro SG and Brasileiro AC: Reference gene selection for qPCR analysis in tomato-bipartite begomovirus interaction and validation in additional tomato-virus pathosystems. PLoS One 10: e0136820, 2015.

34. Zhang X, Ding L and Sandford AJ: Selection of reference genes for gene expression studies in human neutrophils by real-time PCR. BMC Mol Biol 6: 4, 2005.

35. Nelissen K, Smeets K, Mulder M, Hendriks JJ and Ameloot M: Selection of reference genes for gene expression studies in rat oligodendrocytes using quantitative real time PCR. J Neurosci Methods 187: 78-83, 2010.

36. Li H, Chen C, Yao H, Li X, Yang N, Qiao J, Xu K and Zeng L: Identification of suitable reference genes for mRNA studies in bone marrow in a mouse model of hematopoietic stem cell transplantation. Transplant Proc 48: 2826-2832, 2016.

37. Lin $\mathrm{P}$, Lan X, Chen F, Yang Y, Jin Y and Wang A: Reference gene selection for real-time quantitative PCR analysis of the mouse uterus in the peri-implantation period. PLoS One 8: e62462, 2013.

38. Stephens AS, Stephens SR and Morrison NA: Internal control genes for quantitative RT-PCR expression analysis in mouse osteoblasts, osteoclasts and macrophages. BMC Res Notes 4: 410, 2011

39. Klein C, Rutllant $\mathrm{J}$ and Troedsson MH: Expression stability of putative reference genes in equine endometrial, testicular, and conceptus tissues. BMC Res Notes 4: 120, 2011.

40. Valadan R, Hedayatizadeh-Omran A, Alhosseini-Abyazani MN, Amjadi O, Rafiei A, Tehrani M and Alizadeh-Navaei R: Data supporting the design and evaluation of a universal primer pair for pseudogene-free amplification of HPRT1 in real-time PCR. Data Brief 4: 384-389, 2015.

41. ValadanR,AmjadiO,TehraniM,RafieiA,Hedayatizadeh-OmranA and Alizadeh-Navaei R: Pseudogene-free amplification of HPRT1 in quantitative reverse transcriptase polymerase chain reaction. Anal Biochem 485: 46-48, 2015.

42. Zainuddin A, Chua KH, Abdul Rahim N and Makpol S: Effect of experimental treatment on GAPDH mRNA expression as a housekeeping gene in human diploid fibroblasts. BMC Mol Biol 11: 59, 2010

43. Bonefeld BE, Elfving B and Wegener G: Reference genes for normalization: A study of rat brain tissue. Synapse 62: 302-309, 2008. 